\title{
Hematopoiesis in the Fetus and Neonate
}

\author{
ROBERT D. CHRISTENSEN \\ Division of Human Development and Aging. Departments of Pediatrics and Medicine, University of Utah School \\ of Medicine. Salt Lake City, Utah 84132
}

\begin{abstract}
Advances in molecular biology and in hematopoietic progenitor cell culture techniques have produced new tools with which to explore previously enigmatic aspects of developmental hematopoiesis. Studies using these tools are contributing mechanistic understanding to what was previously an entirely descriptive discipline and are generating new insight into the pathophysiology of various cytopenias in fetal and neonatal patients. This review of developmental hematopoiesis will address four related topics: 1 ) ontogeny of blood cells, 2) ontogeny of hematopoietic progenitors, 3) developmental regulation of hematopoietic growth factors, and 4) developmental aspects of interactions between hematopoietic progenitors and hematopoietic growth factors. (Pediatr Res 26:531535,1989 )
\end{abstract}

\section{Abbreviations}

EPO, erythropoietin

CSF, colony-stimulating factor

M-CSF, macrophage colony-stimulating factor

G-CSF, granulocyte colony-stimulating factor

GM-CSF, granulocyte/macrophage colony-stimulating factor

CFU-E, erythroid colony-forming unit

BFU-E, erythroid burst-forming unit

CFU-GM, granulocyte/macrophage colony-forming unit CFU--MIX, multipotent colony-forming unit

\section{ONTOGENY OF BLOOD CELLS}

The anatomic site of blood cell production changes during development from the yolk-sac to the liver to the marrow $(1,2)$. Using abortuses, Kelemen and Janossa (1) identified macrophages as the first-appearing fetal blood cells, present in the liver just before the appearance of hepatic erythropoiesis. Kelemen and Janossa (1) also observed that up to $70 \%$ of circulating blood cells at $4 \mathrm{wk}$ of gestation were macrophages. The first morpho logically recognizable platelets appear in the fetal circulation at 8-9 wh of gestation (2), and circulating platelet concentrations similar to those of adults are achieved by, or before, $18 \mathrm{wk}$ of gestation (3). Neutrophils are the last appearing of the fetal blood cells. In the studies of Keleman et al. (2) and Kelemen and coworkers $(1,2)$ few morphologically recognizable neutrophils were observed in the liver or marrow until about $16 \mathrm{wk}$ of gestation.

All of the early observations regarding fetal hematopoiesis were made using tissues from human abortuses $(1,2)$. However, the recently developed technique of percutaneous umbilical cord

Received June 26, 1989; accepted August 3, 1989.

Correspondence Robert D. Christensen, M.D., Division of Human Development and Aging. University of Utah School of Medicine, 50 North Medical Drive, Salt Lake City, UT 84132 .

Supported by Grant HD-220830 from the NIH

' Presented as part of the American Pediatric Society Presidential State-of-the Ant Symposium on Hematopoietic Growth Factors, Washington. DC, May 2, 1989. blood sampling has supplemented those investigations by providing a source of circulating blood cells from living human fetuses. For instance, Forestier et al. (3) obtained 63 cordocentesis samples from fetuses of $22-23 \mathrm{wk}$ gestation, and compared the leukocyte, erythrocyte, and platelet concentrations in these to umbilical cord blood samples subsequently obtained from the same individuals at the time of term delivery. The fetal leukocyte concentrations were much lower at $22-23$ wk $\left(3.9 \pm 0.2 \times 10^{9}\right.$ cells/L) than at term $\left(11.1 \pm 4.4 \times 10^{9} / \mathrm{L}\right)$. In contrast, erythrocyte concentrations were only slightly lower, and platelet concentrations were identical at 22-23 wk and term. Differential leukocyte counts from these blood samples indicated that only $5+$ $2 \%$ of the fetal leukocytes at 22-23 wk were neutrophils, compared with more than $60 \%$ at term. Thus, blood neutrophil concentrations at $22-23$ wk gestation were only $0.1-0.2 \times 10^{9}$ cells/L compared with $5.0-8.0 \times 10^{9} / \mathrm{L}$ at term.

The very low circulating concentrations of neutrophils in midtrimester human fetuses might suggest that, should such subjects be exposed to bacteria, their defense would be deficient. However, quantification of the neutrophil storage pool is a more direct assessment of the reserve capacity to defend against pyogenic bacteria than is determination of the circulating neutrophil concentration $(4,5)$. The neutrophil storage pool is the sum of all postmitotic neutrophils (segmented neutrophils, band neutrophils, and metamyelocytes) that have not yet been released into the circulation (5). This pool serves as a ready reserve of mature neutrophils, capable of being released into the circulation in response to a need for increased concentrations of neutrophils in the blood or tissues (5). Precise measurement of the size of the neutrophil storage pool is not currently possible in fetal humans, but has been reported in animals. For instance, in rats (4) the size of the neutrophil storage pool increases from essentially no cells at $13 \mathrm{~d}$ gestation (term is about $21 \mathrm{~d}$ ), to $1.2 \pm 0.1$ $\times 10^{6} \mathrm{cells} / \mathrm{g}$ body wt at $19-20 \mathrm{~d}$ gestation, to $1.9 \pm 0.1 \times 10^{6}$ cells/g at term. By 4 wk of age, rats have $4.3 \pm 0.4 \times 10^{6}$ cells $/ g$ and adult rats (like adult dogs and adult humans) have $6-7 \times$ $10^{6}$ stored neutrophils/g $(4,5)$. The very small fetal circulating neutrophil pool and neutrophil storage pool might represent a host-defense limitation for fetuses delivered prematurely, perhaps contributing to the increased incidence of bacterial infection in preterm infants $(6,7)$, as well as to their increased incidence of neutropenia when infected $(8,9)$.

Several developmental studies in animals and man have assessed the changing anatomic location of the neutrophil reserve. In adults the reserve is located entirely within the bone marrow whereas fetal subjects have a substantial fraction of their reserve within the liver and spleen $(4,10,11)$. For instance, fetal rats at $19-20 \mathrm{~d}$ gestation have $>90 \%$ of all stored postmitotic neutrophils located within the liver, whereas $<10 \%$ are in the marrow (4). At term the liver still contains about $60-70 \%$ of the neutrophil reserve, although by 2 wk of age the marrow contains essentially all of the stored neutrophils.

This changing anatomic site of the neutrophil reserve during fetal development may relate to developmentally regulated adhesion-promoting components in the extracellular matrix. For 
example, glycosaminoglycans are present in extracellular matrix of marrow stromal cells (12), and might act to bind hematopoietic growth factors, where they could regulate hematopoiesis locally (13). In support of this postulate, Gordon et al. (14) demonstrated that GM-CSF specifically binds to glycosaminoglycans from adult marrow stromal extracellular matrix. Other adhesion-promoting components of the extracellular matrix might act as attachment sites for hematopoietic cells. For instance, Campbell et al. (15) described a protein termed "hemonectin" in the marrow extracellular matrix of adults which appears to be a lineage-specific attachment molecule for developing granulocytes. Using a murine model, their group recently reported a correlation between presence of hemonectin and the known ontogenic sites of hematopoiesis, migrating from the yolk sac to the liver and spleen and marrow (16). Patel and Lodish (17) and Tsai et al. (17) demonstrated that fibronectin within the extracellular matrix binds BFU-E but not maturing normoblasts. They suggested that modulation of specific adhesion sites might not only regulate the anatomic site of erythropoiesis, but might also regulate release of mature hemic cells from the marrow into the circulation.

In addition to the developmental changes in quantity and anatomic location of stored neutrophils, substantial qualitative changes occur in neutrophils during development. These have recently been reviewed by Hill (19).

\section{ONTOGENY OF HEMATOPOIETIC PROGENITORS}

Hematopoietic progenitors are first observed in the yolk sac. In mice at 7-8 $\mathrm{d}$ of gestation the yolk sac is the major hematopoietic organ, but by $11-13 \mathrm{~d}$ the liver has become the major hematopoietic organ, and after this time the marrow is the major site $(20,21)$. A precise explanation for this switch in the anatomic site of hematopoiesis is not available, however, the developmental regulation of adhesion-promoting components of the extracellular matrix, mentioned previously (12-18), may be of importance.

Experiments of Moore et al. (20) and Metcalf (21) suggest that an intact yolk sac is required for subsequent fetal hematopoiesis, because progenitors from the vascular system of the yolk sac apparently migrate into and colonize the fetal liver. Hematopoietic progenitors in human fetal liver at $5-8$ wk of gestation differ in several respects from the marrow-derived progenitors of adults. These differences include their very rapid cycling rate (22), the constantly and rapidly expanding pool size (23), in vivo differentiation almost exclusively along the erythroid pathway (2), accelerated in vitro maturation time (22), greater sensitivity to erythropoietin (22), and decreased sensitivity to GM-CSF (24).

Unlike the blood of adults, fetal blood contains high concentrations of hematopoietic progenitors. For instance, in healthy adults, Ganser et al. (25) found only $22 \pm 7$ CFU-MIX $/ \mathrm{mL}$ of blood (25). Lipschitz and Udupa (26) and our group (27) reported similar concentrations. Blood concentrations of CFU-GM were measured by McCredie et al. (28), Issaragrisil (29), and our group (30), and all revealed blood concentrations of about 100 (range 30-250) CFU-GM/mL blood in healthy adults. In contrast, umbilical cord blood of term neonates contains very high concentrations of multipotent $(27,29)$ and granulocyte-macrophage progenitors $(2200 / \mathrm{mL}$, range $700-4500 / \mathrm{mL})(30-33)$, and cord blood of extremely preterm fetuses contains two to four times higher concentrations. Similarly, essentially no CFU-E are recovered from the blood of adults, but concentrations of $2000-$ $5000 / \mathrm{mL}$ are generally observed in the cord blood of healthy term neonates $(34,35)$. This high fetal blood concentration of hematopoietic progenitors, however, does not necessarily signify a large total body pool of progenitors. In fact, developmental studies using rats, in which the total body pools of progenitors were obtained by summing the hepatic, splenic, myeloid, and circulating pools, suggesting that the opposite is true. Specifically, at $20 \mathrm{~d}$ gestation rats have only about $1 / 5$ th the CFU-MIX/g body wt (36) and 1/10th CFU-GM/g body wt (37) as do adult animals.

The tritiated thymidine suicide rate of hematopoietic progenitors can be used as an assessment of the relative cell cycle duration $(38,39)$. Thymidine suicide rates of CFU-MIX from marrow of normal adults generally ranges from $0-5 \%(40)$, signifying relative "quiescence" of these progenitors. The tritiated thymidine suicide rate of CFU-GM from the marrow of normal adults is generally $5-20 \%$ (41). In contrast, in umbilical cord blood of term human fetuses, rates of about $50 \%$ are observed for CFU-MIX (27) and CFU-GM (30). Extremely high, perhaps near maximal rates of 70-80\% are observed in CFU-MIX and CFU.GM from fetal blood at 26-28 wk of gestation $(27,30)$. Such rapid cycling of progenitors in the baseline, noninfected, state may impose a limitation in capacity to accelerate neutrophil production during infection. In support of this postulate are studies showing that after incubation with IL-3, CFU-MIX from fetal rats, already cycling very rapidly, failed to undergo any further cell cycle shortening, whereas cycling of CFU-MIX from adult rats greatly increased (29). This latter observation might be of importance if it signifies a limited capacity for increased neutrophil production from progenitors during fetal bacterial infection. In fact, such a limitation has been illustrated in neonatal rats injected with group B streptococci (42). During lethal and nonlethal varieties of infection, neither CFU-GM concentration nor CFU-GM thymidine suicide rates increased, whereas adult rats given a similar inoculum/g body wt experienced a marked increase in CFU-GM concentration and CFU-GM thymidine suicide rate (42). Thus, developmental differences exist in quantity and cell cycle characteristics of hematopoietic progenitors. In adult subjects the relatively large progenitor pool sizes and relative quiescence of progenitors in the steady-state enables substantial acceleration in neutrophil production. A less substantial capacity for acceleration of neutrophil production by fetal subjects might contribute to their higher incidence of neutropenia when infected $(8,9,19)$.

\section{DEVELOPMENTAL REGULATION OF HEMATOPOIETIC GROWTH FACTORS}

Hematopoietic progenitors are unable to survive and proliferate in culture uniess specifically stimulated to do so $(43,44)$. Factors with these stimulatory properties are termed CSF. Listed in Table 1 are the human CSF, their genomic map sites, and their major hematopoietic actions (45-53). In Table 1, factors are divided into those that appear to act directly on hematopoietic progenitors, and those that act indirectly, by stimulating CSF generation.

Although CSF have recentiy become available for study through recombinant DNA techniques, little is known about their developmental regulation. Azoulay et al. (54) assessed fetal mice for expression of M-CSF, G-CSF, GM-CSF, and IL-3. Only M-CSF was detected, both as mRNA and bioactive protein. These findings coincide with the observations of Barak $e t a l$. (55), who demonstrated that although no neutrophils were demonstrated in the liver of human fetuses at 5 wk gestation, liver tissue at that gestation contained progenitors capable of differentiation into neutrophils in vitro. It has been speculated that M-CSF, but not G-CSF, is elaborated, or physiologically active, during the first-trimester of pregnancy, resulting in direction of CFU-GM exclusively into macrophage differentiation.

The placenta has been a common laboratory source of CSF, in the form of placental-conditioned medium $(56,57)$, but whether or not the placenta is a physiologic source of CSF in utero is not clear. Stanley and coworkers (58-60) have demonstrated the presence of the receptor for M-CSF (the c-fms proto oncogene) in human and murine trophoblast, and has demonstrated a 10000 -fold elevation in M-CSF concentration in the uterus of mice during pregnancy. Even though M-CSF activity, measured by RIA and bioassay, is very high in the placenta and 